\title{
Power corrections to the pion transition form factor from higher-twist distribution amplitudes of a photon
}

\author{
Yue-Long Shen, ${ }^{1, *}$ Jing Gao, ${ }^{2,3, \dagger}$ Cai-Dian Lü, ${ }^{2,3,}$ and Yan Miao ${ }^{1, \S}$ \\ ${ }^{1}$ College of Information Science and Engineering, Ocean University of China, Qingdao, \\ Shandong 266100, People's Republic of China \\ ${ }^{2}$ Institute of High Energy Physics, CAS, P.O. Box 918(4), Beijing 100049, People's Republic of China \\ ${ }^{3}$ School of Physics, University of China Academy of Sciences, Beijing 100049, People's Republic of China
}

(Received 31 January 2019; published 13 May 2019)

\begin{abstract}
In this paper, we investigate the power-suppressed contributions from two-particle and three-particle twist-4 light-cone distribution amplitudes (LCDAs) of a photon within the framework of light-cone sum rules. Compared with the leading twist LCDA result, the contribution from three-particle twist-4 LCDAs is not suppressed in the expansion by $1 / Q^{2}$, so that the power corrections considered in this work can give rise to a sizable contribution, especially at the low- $Q^{2}$ region. According to our result, the power-suppressed contributions should be included in the determination of the Gegenbauer moments of pion LCDAs with the pion transition form factor.
\end{abstract}

DOI: 10.1103/PhysRevD.99.096013

\section{INTRODUCTION}

As one of the simplest hard exclusive processes, the pion transition form factor $F_{\gamma^{*} \gamma \rightarrow \pi^{0}}\left(Q^{2}\right)$ at a large momentum transfer is of great importance in exploring the strong interaction dynamics of hadronic reactions in the framework of QCD and to determine the parameters in the lightcone distribution amplitudes (LCDAs) of a pion. It is defined via the matrix element

$$
\begin{aligned}
\left\langle\pi(p)\left|j_{\mu}^{\mathrm{em}}\right| \gamma\left(p^{\prime}\right)\right\rangle & =g_{\mathrm{em}}^{2} \epsilon_{\mu \nu \alpha \beta} q^{\alpha} p^{\beta} \epsilon^{\nu}\left(p^{\prime}\right) F_{\gamma^{*} \gamma \rightarrow \pi^{0}}\left(Q^{2}\right), \\
\epsilon_{0123} & =-1,
\end{aligned}
$$

where $q=p-p^{\prime}, p$, and $p^{\prime}$ refer to the four-momentum of the pion and the on-shell photon, respectively, and the electromagnetic current

$$
j_{\mu}^{\mathrm{em}}=\sum_{q} g_{\mathrm{em}} Q_{q} \bar{q} \gamma_{\mu} q
$$

In the collinear factorization theorem, the pion transition form factor can be factorized into the convolution of the hard kernel and the leading twist pion LCDA at the leading

\footnotetext{
*shenylmeteor@ouc.edu.cn

${ }^{\dagger}$ Corresponding author.

gaojing@ihep.ac.cn

*lucd@ihep.ac.cn

\$782771338@qq.com
}

Published by the American Physical Society under the terms of the Creative Commons Attribution 4.0 International license. Further distribution of this work must maintain attribution to the author(s) and the published article's title, journal citation, and DOI. Funded by SCOAP ${ }^{3}$. power of $1 / Q^{2}$ [1-4], and the hard kernel has been calculated up to the two-loop level [5-8]. At the one-loop level, the factorization formula is written by

$$
\begin{aligned}
F_{\gamma^{*} \gamma \rightarrow \pi^{0}}^{\mathrm{LP}}\left(Q^{2}\right)= & \frac{\sqrt{2}\left(Q_{u}^{2}-Q_{d}^{2}\right) f_{\pi}}{Q^{2}} \int_{0}^{1} d x\left[T_{2}^{(0)}(x)\right. \\
& \left.+T_{2}^{(1), \Delta}(x, \mu)\right] \phi_{\pi}^{\Delta}(x, \mu)+\mathcal{O}\left(\alpha_{s}^{2}\right),
\end{aligned}
$$

where the leading twist pion LCDA is defined as

$$
\begin{aligned}
\left\langle\pi(p)\left|\bar{\xi}(y)[y, 0] \gamma_{\mu} \gamma_{5} \xi(0)\right| 0\right\rangle= & -i f_{\pi} p_{\mu} \int_{0}^{1} d u e^{i u p \cdot y} \phi_{\pi}(u, \mu) \\
& +\mathcal{O}\left(y^{2}\right)
\end{aligned}
$$

and the superscript " $\Delta$ " indicates the scheme to deal with $\gamma_{5}$ in dimensional regularization, which is a subtle problem in QCD loop diagrams [9-15]. Employing the trace technique, the $\gamma_{5}$ ambiguity of dimensional regularization was resolved by adjusting the way of manipulating $\gamma_{5}$ in each diagram to preserve the axial-vector Ward identity [6]. In a recent paper [16], the one-loop calculation is revisited by applying the standard operator product expansion (OPE) technique [17-19] with the evanescent operator(s) [20,21], in both the naive dimensional regularization and HooftVeltman schemes for $\gamma_{5}$ in the $D$-dimensional space. At the one-loop level, it has been shown explicitly that the scheme dependence of the hard kernel and the twist-2 pion LCDA is canceled out precisely, which guarantees the form factor $F_{\gamma^{*} \gamma \rightarrow \pi^{0}}\left(Q^{2}\right)$ to be free from $\gamma_{5}$ ambiguity.

At leading power, the pion transition form factor has also been studied with the transverse momentum-dependent 
(TMD) factorization approach at the one-loop level [22-24], where the joint resummation of the large logarithms $\ln ^{2} k_{\perp}^{2} / Q^{2}$ and $\ln ^{2} x$ was performed in moment and impact-parameter space [25]. The prediction of the joint resummation-improved TMD factorization approach can accommodate the anomalous BABAR measurements [26] of $F_{\gamma^{*} \gamma \rightarrow \pi^{0}}\left(Q^{2}\right)$, which have stimulated intensive theoretical investigations with various phenomenological approaches as well as lattice QCD simulations (see, for instance, [27-29]). In Refs. [30,31], a leading twist pion LCDA with nonvanishing end-point behavior was proposed to explain the anomalous $B A B A R$ data at high $Q^{2}$. Later, it was found that this method is able to be achieved by introducing a sizable nonperturbative soft correction from the TMD pion wave function [32].

To achieve more precise theoretical predictions, power corrections need to be taken into account, especially at low $Q^{2}$. In Refs. [32,33], the soft correction to the leading twist contribution is evaluated with the dispersion approach and found to be crucial to suppress the contributions from higher Gegenbauer moments of the twist-2 pion LCDAs $[25,34]$. Furthermore, the subleading power "hadronic" photon correction can also be taken into account effectively with the dispersion approach. Within this method, the theoretical accuracy for predicting the pion-photon form factor is improved by including the next-to-next-to-leadingorder (NNLO) QCD correction to the twist-2 contribution and the finite-width effect of the unstable vector mesons in the hadronic dispersion relation [35-39]. Another approach to accommodate the contribution from the hadronic photon is to introduce the LCDAs of a photon. In Ref. [16], the QCD factorization of the correlation function for the construction of the light-cone sum rules (LCSRs) for the hadronic photon contribution to the pion-photon form factor is established. Both the hard matching coefficient and the leading twist photon LCDAs are independent of the $\gamma_{5}$ prescription in dimensional regularization, and the nextto-leading logarithmic resummation of the large logarithms was also perform by solving the renormalization group equations in momentum space. The contribution from the twist-4 pion LCDA is also calculated at the tree level in Refs. [16,40]. There is a strong cancellation between this contribution and the contribution from the hadronic structure of the photon, which makes the overall power correction not significant. The LCDAs of a photon, including both the two-particle and three-particle Fock state, have been studied up to the twist-4 level [41]. The higher-twist LCDAs are not suppressed in many processes such as radiative leptonic $B$ meson decay $B \rightarrow \gamma \ell \nu[42,43]$. In this paper, we will investigate the contribution from the full set of the LCDAs of a photon up to twist 4 to the pion transition form factor using the LCSR approach.

The outline of this paper is as follows: In Sec. II, we present the analytic calculation of the pion transition form factor from the higher-twist photon LCDAs within the
LCSR framework. The numerical results and discussions are given in Sec. III. The last section is closing remarks.

\section{POWER CORRECTIONS FROM THE HADRONIC STRUCTURE OF A PHOTON}

All two-particle and three-particle LCDAs of a photon have been defined and classified up to twist 4 , and the expressions of the LCDAs have also been obtained through the conformal expansion in the presence of the background field [41]. To evaluate the power-suppressed contribution to the pion-photon form factor due to the hadronic photon effect, the following correlation function is employed:

$$
\begin{aligned}
G_{\mu}\left(p^{\prime}, q\right) & =\int d^{4} z e^{-i q \cdot z}\left\langle 0\left|\mathrm{~T}\left\{j_{\mu, \perp}^{\mathrm{em}}(z), j_{\pi}(0)\right\}\right| \gamma\left(p^{\prime}\right)\right\rangle \\
& =-g_{\mathrm{em}}^{2} \epsilon_{\mu \nu \alpha \beta}^{\perp} q^{\alpha} p^{\prime \beta} \epsilon^{\nu}\left(p^{\prime}\right) G\left(p^{2}, Q^{2}\right),
\end{aligned}
$$

where the pion-interpolating current $j_{\pi}$ is defined by

$$
j_{\pi}=\frac{1}{\sqrt{2}}\left(\bar{u} \gamma_{5} u-\bar{d} \gamma_{5} d\right) .
$$

The power-counting rule for the external momenta,

$$
|n \cdot p| \sim \bar{n} \cdot p \sim n \cdot p^{\prime} \sim \mathcal{O}\left(\sqrt{Q^{2}}\right),
$$

will be adopted to determine the perturbative matching coefficient entering the factorization formula of $G_{\mu}\left(p^{\prime}, q\right)$. Applying the standard definition for the pion decay constant

$$
\left\langle 0\left|j_{\pi}\right| \pi(p)\right\rangle=-i f_{\pi} \mu_{\pi}(\mu), \quad \mu_{\pi}(\mu) \equiv \frac{m_{\pi}^{2}}{m_{u}(\mu)+m_{d}(\mu)},
$$

we can write down the hadronic dispersion relation of $G\left(p^{2}, Q^{2}\right)$ :

$$
\begin{aligned}
G\left(p^{2}, Q^{2}\right)= & \frac{f_{\pi} \mu_{\pi}(\mu)}{m_{\pi}^{2}-p^{2}-i 0} F_{\gamma^{*} \gamma \rightarrow \pi^{0}}^{\mathrm{NLP}}\left(Q^{2}\right) \\
& +\int_{s_{0}}^{\infty} d s \frac{\rho^{h}\left(s, Q^{2}\right)}{s-p^{2}-i 0} .
\end{aligned}
$$

The form factor $F_{\gamma^{*} \gamma \rightarrow \pi^{0}}^{\mathrm{NLP}}\left(Q^{2}\right)$ will be extracted after the correlation function being calculated by OPE in the deep Euclidean region, and the tree level Feynman diagram of the contribution from two-particle photon LCDAs are plotted in Figure 1. Employing the dispersion relation, subtracting the continuum state contribution with the help of the quark hadron duality assumption, and performing a Borel transformation, the LCSRs for the subleading power contribution to the $\pi^{0} \gamma^{*} \gamma$ form factor are derived as 


$$
\begin{aligned}
F_{\gamma^{*} \gamma \rightarrow \pi^{0}}^{2 \mathrm{PLT}}\left(Q^{2}\right)= & -\frac{\sqrt{2}\left(Q_{u}^{2}-Q_{d}^{2}\right)}{f_{\pi} \mu_{\pi}(\mu) Q^{2}} \chi(\mu)\langle\bar{q} q\rangle(\mu) \\
& \times \int_{0}^{s_{0}} d s \exp \left[-\frac{s-m_{\pi}^{2}}{M^{2}}\right] \\
& \times\left[\rho^{(0)}\left(s, Q^{2}\right)+\frac{\alpha_{s} C_{F}}{4 \pi} \rho^{(1)}\left(s, Q^{2}\right)\right]+\mathcal{O}\left(\alpha_{s}^{2}\right),
\end{aligned}
$$

where the magnetic susceptibility of the quark condensate $\chi(\mu)$ contains the dynamical information of the QCD vacuum, and the spectral functions $\rho^{(0,1)}\left(s, Q^{2}\right)$ can be found in Ref. [16].

Now we will proceed to investigate the contribution from higher-twist LCDAs of a photon. Up to twist 4, the twoparticle LCDAs of a photon are defined as

$$
\begin{aligned}
\left\langle 0\left|\bar{q}(x)[x, 0] \sigma_{\alpha \beta} q(0)\right| \gamma(p)\right\rangle= & i g_{\mathrm{em}} Q_{q}\langle\bar{q} q\rangle(\mu)\left(p_{\beta} \epsilon_{\alpha}-p_{\alpha} \epsilon_{\beta}\right) \int_{0}^{1} d z e^{i z p \cdot x}\left[\chi(\mu) \phi_{\gamma}(z, \mu)\right. \\
& \left.+\frac{x^{2}}{16} \mathbb{A}(z, \mu)\right]+\frac{i}{2} g_{\mathrm{em}} Q_{q} \frac{\langle\bar{q} q\rangle(\mu)}{p \cdot x}\left(x_{\beta} \epsilon_{\alpha}-x_{\alpha} \epsilon_{\beta}\right) \int_{0}^{1} d z e^{i z p \cdot x} h_{\gamma}(z, \mu), \\
\left\langle 0\left|\bar{q}(x)[x, 0] \gamma_{\alpha} q(0)\right| \gamma(p)\right\rangle= & g_{\mathrm{em}} Q_{q} f_{3 \gamma}(\mu) \epsilon_{\alpha} \int_{0}^{1} d z e^{i z p \cdot x} \psi_{\gamma}^{(v)}(z, \mu), \\
\left\langle 0\left|\bar{q}(x)[x, 0] \gamma_{\alpha} \gamma_{5} q(0)\right| \gamma(p)\right\rangle= & \frac{g_{\mathrm{em}} Q_{q} f_{3 \gamma}(\mu)}{4} \varepsilon_{\alpha \beta \rho \tau} p^{\rho} x^{\tau} \epsilon^{\beta} \int_{0}^{1} d z e^{i z p \cdot x} \psi_{\gamma}^{(a)}(z, \mu),
\end{aligned}
$$

where $\psi_{\gamma}^{(v)}(z, \mu), \psi_{\gamma}^{(a)}(z, \mu)$ are twist 3 and $\mathbb{A}(z, \mu), h_{\gamma}(z, \mu)$ are twist 4. Employing the light-cone expansion of the $u, d$-quark propagator and keeping the subleading-power contributions to the correlation function (5) leads to

$$
\begin{aligned}
G_{\mu}\left(p^{\prime}, q\right) & \supset \frac{1}{\sqrt{2}} \int \frac{d^{4} k}{(2 \pi)^{4}} \int d^{4} x e^{i(k-q) \cdot x} \frac{k^{\nu}}{k^{2}} \sum_{q=u, d} \delta_{q} Q_{q} g_{\mathrm{em}}\left\langle 0\left|\bar{q}(x) \sigma_{\mu \nu} \gamma_{5} q(0)\right| \gamma\left(p^{\prime}\right)\right\rangle-(q \leftrightarrow-p) \\
& =\frac{i}{2 \sqrt{2}} \epsilon_{\mu \nu \rho \sigma} \int \frac{d^{4} k}{(2 \pi)^{4}} \frac{k^{\nu}}{k^{2}} \int d^{4} x e^{i(k-q) \cdot x} \sum_{q=u, d} \delta_{q} Q_{q} g_{\mathrm{em}}\left\langle 0\left|\bar{q}(x) \sigma^{\rho \sigma} q(0)\right| \gamma\left(p^{\prime}\right)\right\rangle-(q \leftrightarrow-p),
\end{aligned}
$$

where $\delta_{u}=1, \delta_{d}=-1$. The above equation indicates that only twist- 2 and twist- 4 two-particle LCDAs can contribute to the pion transition form factor in the LCSR approach, which is different from the method based on TMD factorization [44]. Making use of the definitions in Eq. (11), it is straightforward to write down

$$
\begin{aligned}
G_{\mu}^{2 \mathrm{PHT}}(p, q)= & -\frac{g_{\mathrm{em}}^{2}}{4} \epsilon_{\mu \nu \alpha \beta}^{\perp} \varepsilon^{\nu} q^{\alpha} p^{\prime \beta} \frac{Q_{u}^{2}-Q_{d}^{2}}{\sqrt{2} Q^{4}}\langle\bar{q} q\rangle(\mu) \\
& \times \int_{0}^{1} d u\left[\frac{\mathbb{A}(u, \mu)}{(\bar{u}+u r)^{2}}+\frac{\mathbb{A}(u, \mu)}{(u+r \bar{u})^{2}}\right],
\end{aligned}
$$

where the contribution from $h_{\gamma}(z, \mu)$ vanishes due to the antisymmetric structure. The resulting LCSRs for the twoparticle higher-twist hadronic photon corrections to the pion transition form factors can be further derived as follows:

$$
\begin{aligned}
F_{\gamma^{*} \gamma \rightarrow \pi^{0}}^{2 \mathrm{PHT}}\left(Q^{2}\right)= & -\frac{\sqrt{2}\left(Q_{u}^{2}-Q_{d}^{2}\right)}{4 f_{\pi} \mu_{\pi}(\mu)}\langle\bar{q} q\rangle(\mu)\left\{\frac{1}{Q^{2}} \mathbb{A}\left(u_{0}\right) e^{-\frac{s_{0}-m_{\pi}^{2}}{M^{2}}}\right. \\
& \left.+\int_{u_{0}}{ }^{1} \frac{d u}{u^{2}} \frac{1}{M^{2}} \exp \left[-\frac{\bar{u} Q^{2}-u m_{\pi}^{2}}{u M^{2}}\right] \mathbb{A}(u, \mu)\right\},
\end{aligned}
$$

where $u_{0}=Q^{2} /\left(s_{0}+Q^{2}\right)$.
To compute higher-twist three-particle hadronic photon corrections to the pion transition form factors, the definition of three-particle photon LCDA is required. In the Appendix, we collect the definition of three-particle twist-4 photon LCDAs for an incoming photon state. Keeping the one-gluon (photon) part for the light-cone expansion of the quark propagator in the background gluon (photon) field,

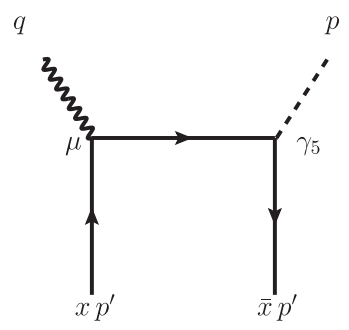

(a)

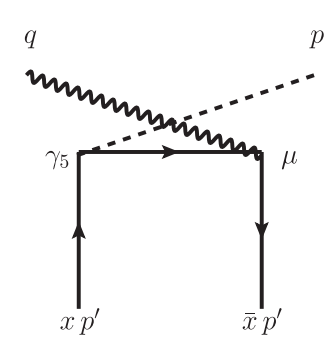

(b)
FIG. 1. Diagrammatical representation of the tree-level contribution to the QCD amplitude $\tilde{G}_{\mu}$ with the contribution from two-particle photon LCDAs. 


$$
\begin{aligned}
& \langle 0|T\{q(x), \bar{q}(0)\}| 0\rangle_{G} \\
& \supset i \int \frac{d^{4} k}{(2 \pi)^{4}} e^{-i k \cdot x} \int_{0}^{1} d u\left[\frac{u x_{\mu} \gamma_{\nu}}{k^{2}}-\frac{\not k \sigma_{\mu \nu}}{2 k^{4}}\right] G^{\mu \nu}(u x) \\
& +i g_{\mathrm{em}} Q_{q} \int_{0}^{\infty} \frac{d^{4} k}{(2 \pi)^{4}} e^{-i k \cdot x} \int_{0}^{1} d u\left[\frac{u x_{\mu} \gamma_{\nu}}{k^{2}}-\frac{\not k \sigma_{\mu \nu}}{2 k^{4}}\right] F^{\mu \nu}(u x),
\end{aligned}
$$

where $G^{\mu \nu}=i\left[D_{\mu}, D_{\nu}\right]$. By evaluating Fig. 2, we obtain

$$
\begin{aligned}
\Pi_{\mu}(p, q) \supset & \frac{1}{2 \sqrt{2}} g_{\mathrm{em}}^{2} \sum_{q} \delta_{q} Q_{q}^{2} \epsilon_{\mu \alpha \rho \lambda} q^{\alpha} \varepsilon^{\rho} p^{\prime \lambda}\langle\bar{q} q\rangle(\mu) \\
& \times \int_{0}^{1} d u \int\left[\mathcal{D} \alpha_{i}\right] \frac{1}{\left[q-\left(\alpha_{q}+\bar{u} \alpha_{g}-1\right) p^{\prime}\right]^{4}} \\
& \times \rho^{3 \mathrm{PHT}}\left(\alpha_{i}, u, \mu\right)-(q \leftrightarrow-p)
\end{aligned}
$$

where

$$
\begin{aligned}
\rho^{3 \mathrm{PHT}}\left(\alpha_{i}, u, \mu\right)= & 2\left\{( 2 u - 1 ) \left[T_{1}\left(\alpha_{i}\right)-T_{2}\left(\alpha_{i}\right)+T_{3}\left(\alpha_{i}\right)\right.\right. \\
& \left.+T_{4}\left(\alpha_{i}\right)-\tilde{S}\left(\alpha_{i}\right)+T_{4 \gamma}\left(\alpha_{i}\right)\right] \\
& +S\left(\alpha_{i}, \mu\right)+S_{\gamma}\left(\alpha_{i}, \mu\right)+T_{2}\left(\alpha_{i}, \mu\right) \\
& \left.-T_{1}\left(\alpha_{i}, \mu\right)\right\}
\end{aligned}
$$

and the integration measure is defined as

$$
\int\left[\mathcal{D} \alpha_{i}\right] \equiv \int_{0}^{1} d \alpha_{q} \int_{0}^{1} d \alpha_{\bar{q}} \int_{0}^{1} d \alpha_{g} \delta\left(1-\alpha_{q}-\alpha_{\bar{q}}-\alpha_{g}\right) .
$$

Taking advantage of quark-hadron duality, we arrive at the LCSRs of the contribution from three-particle photon LCDAs:

$$
\begin{aligned}
F_{\gamma^{*} \gamma \rightarrow \pi^{0}}^{3 \mathrm{PHT}}\left(Q^{2}\right)= & -\frac{\sqrt{2}\left(Q_{u}^{2}-Q_{d}^{2}\right)}{2 f_{\pi} \mu_{\pi}(\mu)}\langle\bar{q} q\rangle(\mu) \frac{1}{Q^{2}}\left\{\int_{0}^{s_{0} /\left(s_{0}+Q^{2}\right)} d \alpha_{q} \int_{s_{0} /\left(s_{0}+Q^{2}\right)-\alpha_{q}}^{1-\alpha_{q}} \frac{d \alpha_{g}}{\alpha_{g}} \rho^{3 \mathrm{PHT}}\left(\alpha_{q}, \alpha_{g}, \alpha_{\bar{q}}=1-\alpha_{q}-\alpha_{g}, u_{s_{0}}, \mu\right) e^{-\frac{s_{0}-m_{\pi}^{2}}{M^{2}}}\right. \\
& \left.+\frac{1}{M^{2}} \int_{0}^{s_{0}} d s e^{-\frac{s-m_{\pi}^{2}}{M^{2}}} \int_{0}^{s /\left(s+Q^{2}\right)} d \alpha_{q} \int_{s /\left(s+Q^{2}\right)-\alpha_{q}}^{1-\alpha_{q}} \frac{d \alpha_{g}}{\alpha_{g}} \rho^{3 \mathrm{PHT}}\left(\alpha_{q}, \alpha_{g}, \alpha_{\bar{q}}=1-\alpha_{q}-\alpha_{g}, u_{s}, \mu\right)\right\}
\end{aligned}
$$

where $u_{s}=\left[s /\left(s+Q^{2}\right)-\alpha_{q}\right] / \alpha_{g}$. The overall higher-twist photon LCDA contribution is written by

$$
F_{\gamma^{*} \gamma \rightarrow \pi^{0}}^{\mathrm{HT}}\left(Q^{2}\right)=F_{\gamma^{*} \gamma \rightarrow \pi^{0}}^{2 \mathrm{PHT}}\left(Q^{2}\right)+F_{\gamma^{*} \gamma \rightarrow \pi^{0}}^{3 \mathrm{PHT}}\left(Q^{2}\right) .
$$

Now we discuss the power behavior of our results. The power-counting scheme for the sum rule parameters are given below:

$$
s_{0} \sim M^{2} \sim \mathcal{O}\left(\Lambda^{2}\right), \quad \bar{u}_{0} \sim \mathcal{O}\left(\Lambda^{2} / Q^{2}\right) .
$$

Employing Eq. (21), one can obtain that the contribution from the leading twist LCDA of photon is suppressed by a

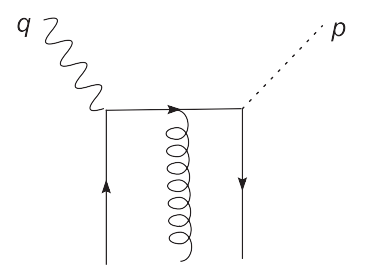

(a)

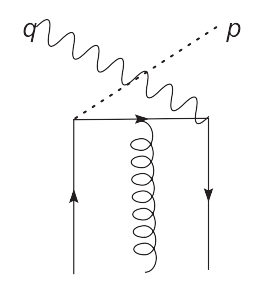

(b)
FIG. 2. Diagrammatical representation of the tree-level contribution to the three-particle photon LCDAs. factor of $\Lambda^{2} / Q^{2}$ [16] compared with the LP contribution. The higher-twist contributions are conjectured to be also suppressed by only one power of $\Lambda^{2} / Q^{2}$ due to the absent correspondence between the twist counting and the largemomentum expansion [32]. For the contribution from two-particle twist-4 LCDAs of a photon, the result in Eq. (14) is suppressed by $\Lambda^{4} / Q^{4}$ compared with the LP contribution, as the power of twist-4 photon LCDAs is suppressed with respect to the leading twist one, while for the contribution from three-particle twist-4 LCDAs in Eq. (19), the scaling of $\alpha_{q}$ is $\mathcal{O}\left(\Lambda^{2} / Q^{2}\right)$, and $\alpha_{g}$ is $\mathcal{O}(1)$. Although there is an overall factor $1 / Q^{2}$, the result is suppressed only by $\Lambda^{2} / Q^{2}$, for the spectral function $\rho^{3 \mathrm{PHT}}$ is not suppressed at the end-point region. This result confirms the conjecture in Ref. [32].

\section{NUMERICAL ANALYSIS}

In the following, we explore the phenomenological consequences of the hadronic photon correction to the pion-photon form factor, and the most important input is the LCDAs of the photon. The models of twist-4 LCDAs of the photon used in this paper are written by 


$$
\begin{aligned}
\mathbb{A}(z, \mu)= & 40 z^{2} \bar{z}^{2}\left[3 \kappa(\mu)-\kappa^{+}(\mu)+1\right]+8\left[\zeta_{2}^{+}(\mu)-3 \zeta_{2}(\mu)\right][z \bar{z}(2+13 z \bar{z}) \\
& \left.+2 z^{3}\left(10-15 z+6 z^{2}\right) \ln z+2 \bar{z}^{3}\left(10-15 \bar{z}+6 \bar{z}^{2}\right) \ln \bar{z}\right], \\
h_{\gamma}(z, \mu)= & -10\left(1+2 \kappa^{+}(\mu)\right) C_{2}^{1 / 2}(2 z-1), \\
S\left(\alpha_{i}, \mu\right)= & 30 \alpha_{g}^{2}\left\{\left(\kappa(\mu)+\kappa^{+}(\mu)\right)\left(1-\alpha_{g}\right)+\left(\zeta_{1}+\zeta_{1}^{+}\right)\left(1-\alpha_{g}\right)\left(1-2 \alpha_{g}\right)+\zeta_{2}(\mu)\left[3\left(\alpha_{\bar{q}}-\alpha_{q}\right)^{2}-\alpha_{g}\left(1-\alpha_{g}\right)\right]\right\}, \\
\tilde{S}\left(\alpha_{i}, \mu\right)= & -30 \alpha_{g}^{2}\left\{\left(\kappa(\mu)-\kappa^{+}(\mu)\right)\left(1-\alpha_{g}\right)+\left(\zeta_{1}-\zeta_{1}^{+}\right)\left(1-\alpha_{g}\right)\left(1-2 \alpha_{g}\right)+\zeta_{2}(\mu)\left[3\left(\alpha_{\bar{q}}-\alpha_{q}\right)^{2}-\alpha_{g}\left(1-\alpha_{g}\right)\right]\right\}, \\
S_{\gamma}\left(\alpha_{i}, \mu\right)= & 60 \alpha_{g}^{2}\left(\alpha_{q}+\alpha_{\bar{q}}\right)\left[4-7\left(\alpha_{\bar{q}}+\alpha_{q}\right)\right], \\
T_{1}\left(\alpha_{i}, \mu\right)= & -120\left(3 \zeta_{2}(\mu)+\zeta_{2}^{+}(\mu)\right)\left(\alpha_{\bar{q}}-\alpha_{q}\right) \alpha_{\bar{q}} \alpha_{q} \alpha_{g}, \\
T_{2}\left(\alpha_{i}, \mu\right)= & 30 \alpha_{g}^{2}\left(\alpha_{\bar{q}}-\alpha_{q}\right)\left[\left(\kappa(\mu)-\kappa^{+}(\mu)\right)+\left(\zeta_{1}(\mu)-\zeta_{1}^{+}(\mu)\right)\left(1-2 \alpha_{g}\right)+\zeta_{2}(\mu)\left(3-4 \alpha_{g}\right)\right], \\
T_{3}\left(\alpha_{i}, \mu\right)= & -120\left(3 \zeta_{2}(\mu)-\zeta_{2}^{+}(\mu)\right)\left(\alpha_{\bar{q}}-\alpha_{q}\right) \alpha_{\bar{q}} \alpha_{q} \alpha_{g}, \\
T_{4}\left(\alpha_{i}, \mu\right)= & 30 \alpha_{g}^{2}\left(\alpha_{\bar{q}}-\alpha_{q}\right)\left[\left(\kappa(\mu)+\kappa^{+}(\mu)\right)+\left(\zeta_{1}(\mu)+\zeta_{1}^{+}(\mu)\right)\left(1-2 \alpha_{g}\right)+\zeta_{2}(\mu)\left(3-4 \alpha_{g}\right)\right], \\
T_{4}^{\gamma}\left(\alpha_{i}, \mu\right)= & 60 \alpha_{g}^{2}\left(\alpha_{q}-\alpha_{\bar{q}}\right)\left[4-7\left(\alpha_{\bar{q}}+\alpha_{q}\right)\right] .
\end{aligned}
$$

In the above equations, the conformal expansions of the photon LCDAs have been truncated up to the next-toleading conformal spin. Because of the Ferrara-GrilloParisi-Gatto theorem [45], these parameters satisfy the following relations:

$$
\zeta_{1}(\mu)+11 \zeta_{2}(\mu)-2 \zeta_{2}^{+}(\mu)=\frac{7}{2} .
$$

The scale evolution of the nonperturbative parameters is given by

$$
\begin{aligned}
\kappa^{+}(\mu) & =\left(\frac{\alpha_{s}(\mu)}{\alpha_{s}\left(\mu_{0}\right)}\right)^{\left(\gamma^{+}-\gamma_{q \bar{q}}\right) / \beta_{0}} \kappa^{+}\left(\mu_{0}\right), \\
\kappa(\mu) & =\left(\frac{\alpha_{s}(\mu)}{\alpha_{s}\left(\mu_{0}\right)}\right)^{\left(\gamma^{-}-\gamma_{q \bar{q}}\right) / \beta_{0}} \kappa\left(\mu_{0}\right), \\
\zeta_{1}(\mu) & =\left(\frac{\alpha_{s}(\mu)}{\alpha_{s}\left(\mu_{0}\right)}\right)^{\left(\gamma_{\left.Q^{(1)}-\gamma_{q \bar{q}}\right) / \beta_{0}}\right.} \zeta_{1}\left(\mu_{0}\right), \\
\zeta_{1}^{+}(\mu) & =\left(\frac{\alpha_{s}(\mu)}{\alpha_{s}\left(\mu_{0}\right)}\right)^{\left(\gamma_{\left.Q^{(5)}-\gamma_{q \bar{q}}\right) / \beta_{0}}\right.} \zeta_{1}^{+}\left(\mu_{0}\right), \\
\zeta_{2}^{+}(\mu) & =\left(\frac{\alpha_{s}(\mu)}{\alpha_{s}\left(\mu_{0}\right)}\right)^{\left(\gamma_{\left.Q^{(3)}-\gamma_{q \bar{q}}\right) / \beta_{0}}\right.} \zeta_{2}^{+}\left(\mu_{0}\right),
\end{aligned}
$$

where the anomalous dimensions at one loop read [41]

$$
\begin{gathered}
\gamma^{+}=3 C_{A}-\frac{5}{3} C_{F}, \quad \gamma^{-}=4 C_{A}-3 C_{F}, \quad \gamma_{q \bar{q}}=-3 C_{F}, \\
\gamma_{Q^{(1)}}=\frac{11}{2} C_{A}-3 C_{F}, \quad \gamma_{Q^{(3)}}=\frac{13}{3} C_{F}, \quad \gamma_{Q^{(5)}}=5 C_{A}-\frac{8}{3} C_{F} .
\end{gathered}
$$

The numerical values of the input parameters entering the photon LCDAs up to twist 4 are collected in Table I, where for the estimates of the twist-4 parameters from QCD sum rules [46] 100\% uncertainties are assigned.

Now we are in the position to investigate the phenomenological significance of the contribution from highertwist photon LCDAs. For the factorization scale in the evaluation of the contribution of higher-twist photon LCDAs, we will take the value $\mu^{2}=\langle x\rangle M^{2}+\langle\bar{x}\rangle Q^{2}$ as widely employed in the sum rule calculations [32]. The Borel mass $M^{2}$ and the threshold parameter $s_{0}$ can be determined by applying the standard strategies described in Refs. [48,49]:

$$
M^{2}=(1.25 \pm 0.50) \mathrm{GeV}^{2}, \quad s_{0}=(0.70 \pm 0.20) \mathrm{GeV}^{2}
$$

where the variation ranges of these parameters are set to be large to allow sufficient theoretical uncertainty. It has been checked that the Borel mass and threshold parameter dependence of the contribution of higher-twist photon LCDAs is mild in the intervals in Eq. (26). In Fig. 3, the $Q^{2}$ dependence of the relevant power-suppressed contributions is presented. Compared with the contribution from the leading twist photon LCDA, the two-particle twist- 4 contribution is obviously suppressed, as the curve declines more quickly and approaches zero at large $Q^{2}$, while for the contribution from three-particle twist-4 LCDAs of a photon, the result is comparable with that from leading twist photon LCDA, as they are at the same

TABLE I. The numerical values of the nonperturbative parameters entering the photon LCDAs at the scale $\mu_{0}=1.0 \mathrm{GeV}$ [41,47].

\begin{tabular}{lccccccc}
\hline \hline$\chi\left(\mu_{0}\right)$ & $\langle\bar{q} q\rangle\left(\mu_{0}\right)$ & $b_{2}\left(\mu_{0}\right)$ & $\kappa\left(\mu_{0}\right)$ & $\kappa^{+}\left(\mu_{0}\right)$ & $\zeta_{1}\left(\mu_{0}\right)$ & $\zeta_{1}^{+}\left(\mu_{0}\right)$ & $\zeta_{2}^{+}\left(\mu_{0}\right)$ \\
\hline \hline$(3.15 \pm 0.3) \mathrm{GeV}^{-2}$ & $-\left(246_{-19}^{+28} \mathrm{MeV}\right)^{3}$ & $0.07 \pm 0.07$ & $0.2 \pm 0.2$ & 0 & $0.4 \pm 0.4$ & 0 & 0 \\
\hline \hline
\end{tabular}




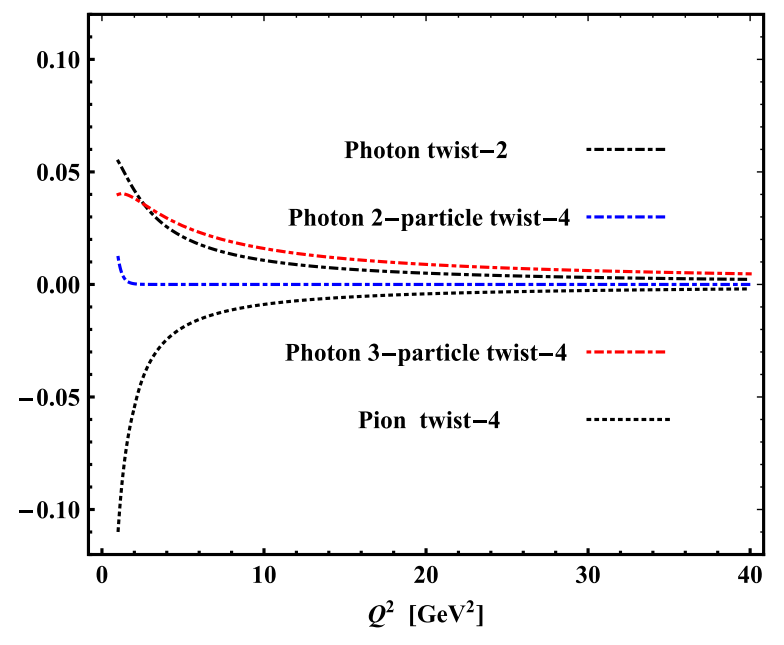

FIG. 3. Comparison of the power-suppressed contribution to pion-photon form factor $Q^{2} F_{\gamma^{*} \gamma \rightarrow \pi^{0}}\left(Q^{2}\right)$ from different sources.

power. As mentioned in Ref. [16], there exists a strong cancellation effect between the contribution from the leading twist photon LCDA and the twist-4 pion LCDA; thus, the overall power correction is mainly from the contribution from twist-4 LCDAs of the photon.

To obtain the total result of the photon-pion form factor, we will need to specify the nonperturbative models for the twist-2 pion LCDA. In general, it is expanded in terms of Gegenbauer polynomials

$$
\phi_{\pi}(x, \mu)=6 x \bar{x} \sum_{n=0}^{\infty} a_{n}(\mu) C_{n}^{3 / 2}(2 x-1),
$$

where the Gegenbauer moments $a_{n}$ can be determined by the calculation with QCD sum rules or lattice simulation or by fitting the experimental data. Following Ref. [16], we take advantage of the Chernyak-Zhitnitsky (CZ) model [50], the Bakulev-Mikhailov-Stefanis (BMS) model [51], the platykurtic model (PK) [52], the Khodjamirian-MannelOffen-Wang (KMOW) model [53], and the holographic model [54] for comparison. The Gegenbauer coefficients in the BMS model and the PK model are computed from the QCD sum rules with nonlocal condensates, the first and second nontrivial Gegenbauer moments of the KMOW model are determined by comparing the LCSR predictions for the pion electromagnetic form factor with the experimental data at intermediate $Q^{2}$, and the holographic model of the twist-2 pion LCDA is motivated by the AdS/QCD correspondence. We collect the values of the Gegenbauer

TABLE II. The numerical values of Gegenbauer moments $a_{2}$ and $a_{4}$ in leading twist pion LCDA.

\begin{tabular}{|c|c|c|c|c|c|}
\hline Models & $\mathrm{CZ}$ & BMS & KMOW & Holographic & Platykurtic \\
\hline$a_{2}(1 \mathrm{GeV})$ & 0.5 & $0.20_{-0.08}^{+0.07}$ & $0.17 \pm 0.08$ & 0.15 & 0.08 \\
\hline$a_{4}(1 \mathrm{GeV})$ & $0-$ & $-0.15_{-0.09}^{+0.10}$ & $0.06 \pm 0.10$ & 0.06 & -0.02 \\
\hline
\end{tabular}

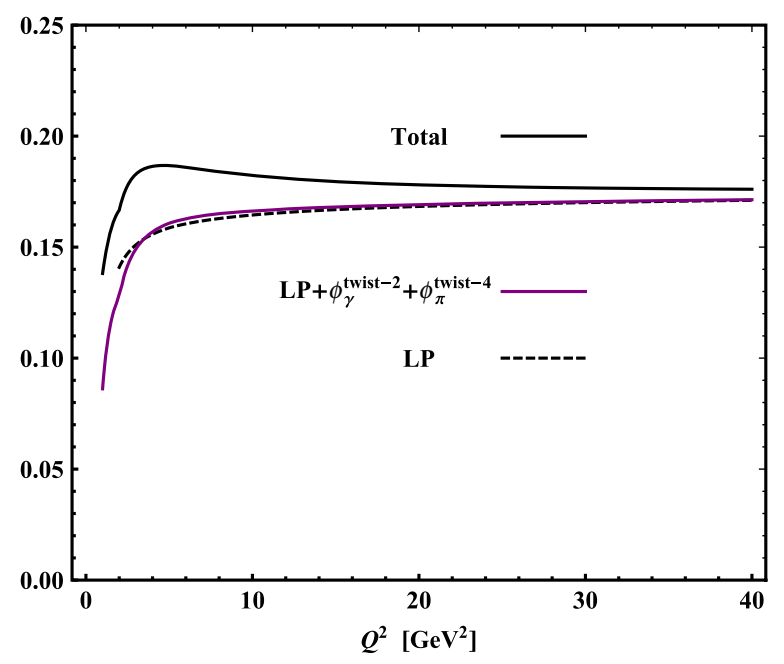

FIG. 4. Total result of the pion-photon form factors $Q^{2} F_{\gamma^{*} \gamma \rightarrow \pi^{0}}\left(Q^{2}\right)$ after including power corrections.

moments in different models in Table II. The total results including power-suppressed contributions are shown in Fig. 4, where the BMS model is employed. It can be seen that the higher-power photon LCDAs manifestly modify the LP result especially at the "small" $Q^{2}$ region. We note that the photon LCSR employed in this paper is valid when $Q^{2} \gg 2 \mathrm{GeV}^{2}$; thus, the prediction of $F_{\gamma^{*} \gamma \rightarrow \pi^{0}}\left(Q^{2}\right)$ should not be taken seriously below $2 \mathrm{GeV}^{2}$. The model dependence of the pion-photon form factor on the leading twist pion LCDA is displayed in Fig. 5. As the contribution from the higher-twist photon LCDA enhances the form factors

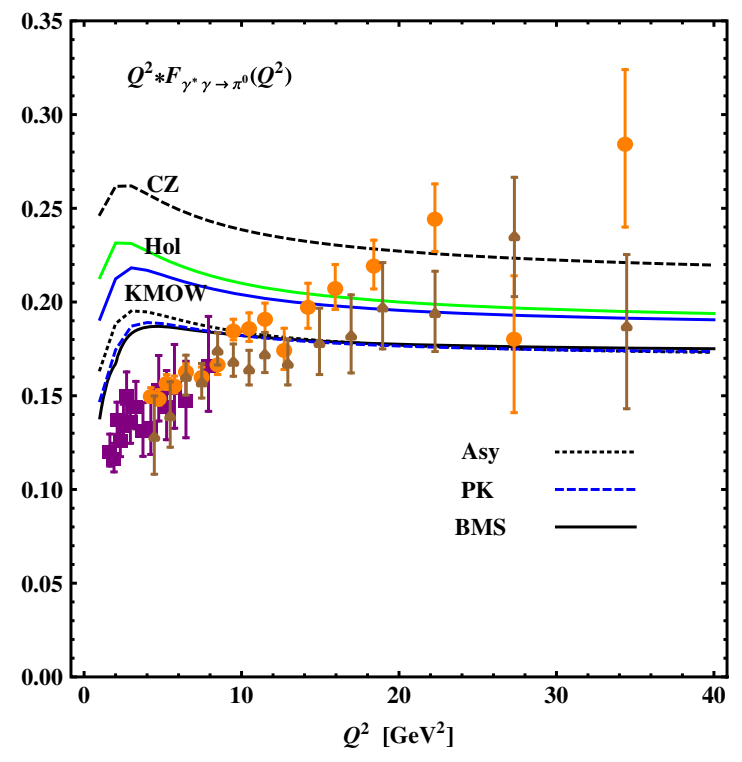

FIG. 5. Total result of the pion-photon form factors $Q^{2} F_{\gamma^{*} \gamma \rightarrow \pi^{0}}\left(Q^{2}\right)$ with different models of leading twist pion LCDA. Points from CLEO [55] (purple squares), BABAR [26] (orange circles), and Belle [56] (brown spades) are displayed here. 


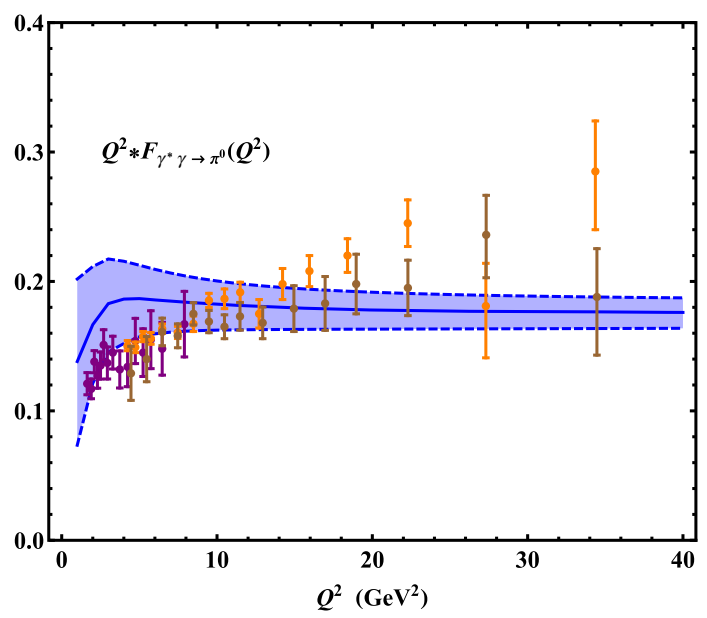

(a)

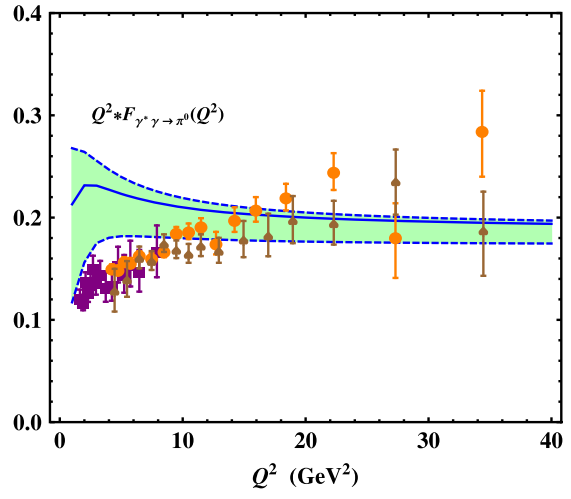

(b)

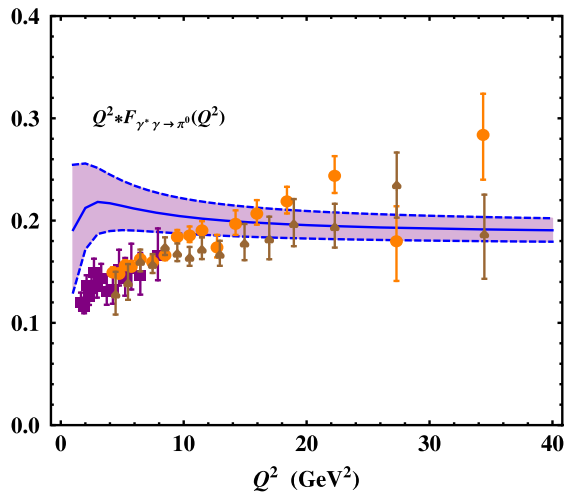

(c)

FIG. 6. Comparison between the theoretical predictions in this paper and the experimental data. Points from CLEO [55] (purple squares), BABAR [26] (orange circles), and Belle [56] (brown spades) are displayed here. Diagrams (a)-(c) correspond to the BMS model, holographic model, and KMOW model of pion LCDA employed, respectively.

significantly, the prediction from every model cannot match the experimental data at $Q^{2}<10 \mathrm{GeV}^{2}$. This result is inconsistent with the predictions from the dispersion approach [35-39], where the BMS and PK models of pion LCDA work well. This discrepancy is not a surprise, because the power-suppressed contributions considered in both approaches are not from a systematic study based on the effective theory, and what is omitted is not clear. Our result indicates that there exist significant powersuppressed contributions, and they should not be neglected in phenomenological studies. Meanwhile, we cannot draw the conclusion that the models mentioned in this paper should be ruled out, because in our study the QCD corrections are not included, and contributions from the pion and photon LCDA with a twist higher than 4 are not considered, let alone the unknown power-suppressed contributions. Thus, in the present paper, we aim at shedding light on the importance of the power corrections, and more efforts must be devoted to the study of power-suppressed contributions to obtain a more accurate prediction.

We present our final predictions for $Q^{2} F_{\gamma^{*} \gamma \rightarrow \pi^{0}}\left(Q^{2}\right)$ with both the LP contribution and power corrections included in
Fig. 6, where the combined theory uncertainties are due to the variations of the input parameters $a_{2}, a_{4}$ of pion LCDA, $\xi,\langle\bar{q} q\rangle, b_{2}$ in twist-2 photon LCDAs, $\kappa, \zeta_{1}, \zeta_{2}$ in twist-4 photon LCDAs, quark mass, and factorization scale, etc. Diagrams (a)-(c) in Fig. 6 correspond to the BMS model, holographic model, and KMOW model of pion LCDA, respectively. Among all the parameters, the most important uncertainty comes from the shape parameters $a_{2}, a_{4}$ of the leading twist pion LCDA, which means the pion transition form factor is still sensitive to the Gegenbauer moments of leading twist pion LCDA after the power-suppressed contributions considered. Thus, the photon-pion transition process provides a good platform to determine the parameters in the LCDAs of a pion, which can also be compared with future lattice simulations with the help of the quasiparton distribution amplitude [57,58].

\section{CLOSING REMARK}

In this paper, we performed a study on the powersuppressed contributions from higher-twist LCDAs of a photon within the LCSR. The twist-3 LCDAs cannot 
contribute for their Lorentz structures; thus, the contributions from two-particle and three-particle twist-4 LCDAs of a photon are considered in this work. According to the power analysis, the three-particle twist- 4 contribution is not suppressed compared with the leading twist photon LCDA result, so that the power corrections considered in this work can give rise to a sizable contribution, especially at the low $Q^{2}$ region. In addition, there exists a strong cancellation between the contribution from the leading twist photon LCDA and the twist-4 pion LCDA, and the importance of the twist-4 photon LCDAs is further highlighted. The numerical result also confirms that, after including power corrections, the predicted $Q^{2} F_{\gamma^{*} \gamma \rightarrow \pi^{0}}\left(Q^{2}\right)$ is significantly enhanced especially at the low $Q^{2}$ region; thus, the powersuppressed contributions should be included in the determination of the Gegenbauer moments of pion LCDAs. Note that, for the higher-twist photon LCDAs contribution, we presented only a tree-level calculation, and the NLO QCD corrections which might modify the current result to some extent and stabilize the factorization scale dependence are not considered. Furthermore, other powersuppressed contributions are also absent in the present study, and a more systematic study based on the effective theory is necessary for a thorough understanding of the NLP corrections to the pion transition form factor, which can be checked by the (potentially) more accurate experimental measurements at the BEPCII collider and the SuperKEKB accelerator.

\section{ACKNOWLEDGMENTS}

We thank S. V. Mikhailov and N. G. Stefanis for valuable comments. This work is supported in part by the National Natural Science Foundation of China (NSFC) with Grants No. 11521505 and No. 11621131001. C. D. Lü expresses special thanks to the Mainz Institute for Theoretical Physics (MITP) for its hospitality and support.

\section{APPENDIX: DEFINITION OF THREE-PARTICLE TWIST-4 LCDAS OF A PHOTON}

In the following, we present the definition of the threeparticle photon LCDAs up to twist 4:

$$
\begin{aligned}
& \left\langle 0\left|\bar{q}(x) g_{s} G_{\alpha \beta}(u x) q(0)\right| \gamma(p)\right\rangle \\
& =i g_{\mathrm{em}} Q_{q}\langle\bar{q} q\rangle(\mu)\left(p_{\beta} \epsilon_{\alpha}-p_{\alpha} \epsilon_{\beta}\right) \\
& \times \int\left[\mathcal{D} \alpha_{i}\right] e^{i\left(\alpha_{q}+\bar{u} \alpha_{g}-1\right) p \cdot x} S\left(\alpha_{i}, \mu\right), \\
& \left\langle 0\left|\bar{q}(x) g_{s} \tilde{G}_{\alpha \beta}(u x) i \gamma_{5} q(0)\right| \gamma(p)\right\rangle \\
& =i g_{\mathrm{em}} Q_{q}\langle\bar{q} q\rangle(\mu)\left(p_{\beta} \epsilon_{\alpha}-p_{\alpha} \epsilon_{\beta}\right) \\
& \times \int\left[D \alpha_{i}\right] e^{i\left(\alpha_{q}+\bar{u} \alpha_{g}-1\right) p \cdot x} \tilde{S}\left(\alpha_{i}, \mu\right), \\
& \left\langle 0\left|\bar{q}(x) g_{s} \tilde{G}_{\alpha \beta}(u x) \gamma_{\rho} \gamma_{5} q(0)\right| \gamma(p)\right\rangle \\
& =-g_{\mathrm{em}} Q_{q} f_{3 \gamma}(\mu) p_{\rho}\left(p_{\beta} \epsilon_{\alpha}-p_{\alpha} \epsilon_{\beta}\right) \\
& \times \int\left[\mathcal{D} \alpha_{i}\right] e^{i\left(\alpha_{q}+\bar{u} \alpha_{g}-1\right) p \cdot x} A\left(\alpha_{i}, \mu\right), \\
& \left\langle 0\left|\bar{q}(x) g_{s} G_{\alpha \beta}(u x) i \gamma_{\rho} q(0)\right| \gamma(p)\right\rangle \\
& =g_{\mathrm{em}} Q_{q} f_{3 \gamma}(\mu) p_{\rho}\left(p_{\beta} \epsilon_{\alpha}-p_{\alpha} \epsilon_{\beta}\right) \\
& \times \int\left[\mathcal{D} \alpha_{i}\right] e^{i\left(\alpha_{q}+\bar{u} \alpha_{g}-1\right) p \cdot x} V\left(\alpha_{i}, \mu\right) \text {, } \\
& \left\langle 0\left|\bar{q}(x) g_{\mathrm{em}} Q_{q} F_{\alpha \beta}(u x) q(0)\right| \gamma(p)\right\rangle \\
& =i g_{\mathrm{em}} Q_{q}\langle\bar{q} q\rangle(\mu)\left(p_{\beta} \epsilon_{\alpha}-p_{\alpha} \epsilon_{\beta}\right) \\
& \times \int\left[\mathcal{D} \alpha_{i}\right] e^{i\left(\alpha_{q}+\bar{u} \alpha_{g}-1\right) p \cdot x} S_{\gamma}\left(\alpha_{i}, \mu\right),
\end{aligned}
$$

$$
\begin{aligned}
\left\langle 0\left|\bar{q}(x) \sigma_{\rho \tau} g_{s} G_{\alpha \beta}(u x) q(0)\right| \gamma(p)\right\rangle= & -g_{\mathrm{em}} Q_{q}\langle\bar{q} q\rangle(\mu)\left[p_{\rho} \epsilon_{\alpha} g_{\tau \beta}^{\perp}-p_{\tau} \epsilon_{\alpha} g_{\rho \beta}^{\perp}-(\alpha \leftrightarrow \beta)\right] \int\left[\mathcal{D} \alpha_{i}\right] e^{i\left(\alpha_{q}+\bar{u} \alpha_{g}-1\right) p \cdot x} T_{1}\left(\alpha_{i}, \mu\right) \\
& -g_{\mathrm{em}} Q_{q}\langle\bar{q} q\rangle(\mu)\left[p_{\alpha} \epsilon_{\rho} g_{\tau \beta}^{\perp}-p_{\beta} \epsilon_{\rho} g_{\tau \alpha}^{\perp}-(\rho \leftrightarrow \tau)\right] \int\left[\mathcal{D} \alpha_{i}\right] e^{i\left(\alpha_{q}+\bar{u} \alpha_{g}-1\right) p \cdot x} T_{2}\left(\alpha_{i}, \mu\right) \\
& -g_{\mathrm{em}} Q_{q}\langle\bar{q} q\rangle(\mu) \frac{\left(p_{\alpha} x_{\beta}-p_{\beta} x_{\alpha}\right)\left(p_{\rho} \epsilon_{\tau}-p_{\tau} \epsilon_{\rho}\right)}{p \cdot x} \int\left[\mathcal{D} \alpha_{i}\right] e^{i\left(\alpha_{q}+\bar{u} \alpha_{g}-1\right) p \cdot x} T_{3}\left(\alpha_{i}, \mu\right) \\
& -g_{\mathrm{em}} Q_{q}\langle\bar{q} q\rangle(\mu) \frac{\left(p_{\rho} x_{\tau}-p_{\tau} x_{\rho}\right)\left(p_{\alpha} \epsilon_{\beta}-p_{\beta} \epsilon_{\alpha}\right)}{p \cdot x} \int\left[\mathcal{D} \alpha_{i}\right] e^{i\left(\alpha_{q}+\bar{u} \alpha_{g}-1\right) p \cdot x} T_{4}\left(\alpha_{i}, \mu\right)
\end{aligned}
$$


Note that we have employed the following notations for the dual field strength tensor and the integration measure:

$$
\tilde{G}_{\alpha \beta}=\frac{1}{2} \varepsilon_{\alpha \beta \rho \tau} G^{\rho \tau}, \quad \int\left[\mathcal{D} \alpha_{i}\right] \equiv \int_{0}^{1} d \alpha_{q} \int_{0}^{1} d \alpha_{\bar{q}} \int_{0}^{1} d \alpha_{g} \delta\left(1-\alpha_{q}-\alpha_{\bar{q}}-\alpha_{g}\right) .
$$

[1] G. P. Lepage and S. J. Brodsky, Phys. Rev. D 22, 2157 (1980).

[2] A. V. Efremov and A. V. Radyushkin, Phys. Lett. 94B, 245 (1980).

[3] A. Duncan and A. H. Müeller, Phys. Lett. 90B, 159 (1980).

[4] I. Z. Rothstein, Phys. Rev. D 70, 054024 (2004).

[5] F. del Aguila and M. K. Chase, Nucl. Phys. B193, 517 (1981).

[6] E. Braaten, Phys. Rev. D 28, 524 (1983).

[7] E. P. Kadantseva, S. V. Mikhailov, and A. V. Radyushkin, Yad. Fiz. 44, 507 (1986) [Sov. J. Nucl. Phys. 44, 326 (1986)].

[8] B. Melic, D. Müeller, and K. Passek-Kumericki, Phys. Rev. D 68, 014013 (2003).

[9] G. Bonneau, Int. J. Mod. Phys. A 05, 3831 (1990).

[10] J. C. Collins, Renormalization: An Introduction to Renormalization, The Renormalization Group, and the Operator Product Expansion (Cambridge University Press, Cambridge, England, 1984).

[11] S. A. Larin, Phys. Lett. B 303, 113 (1993).

[12] C. P. Martin and D. Sanchez-Ruiz, Nucl. Phys. B572, 387 (2000).

[13] F. Jegerlehner, Eur. Phys. J. C 18, 673 (2001).

[14] S. Moch, J. A. M. Vermaseren, and A. Vogt, Phys. Lett. B 748, 432 (2015).

[15] D. Gutirrez-Reyes, I. Scimemi, and A. A. Vladimirov, Phys. Lett. B 769, 84 (2017).

[16] Y. M. Wang and Y. L. Shen, J. High Energy Phys. 12 (2017) 037.

[17] M. Beneke, Y. Kiyo, and D. S. Yang, Nucl. Phys. B692, 232 (2004).

[18] M. Beneke and D. Yang, Nucl. Phys. B736, 34 (2006).

[19] M. Beneke and S. Jager, Nucl. Phys. B751, 160 (2006).

[20] M. J. Dugan and B. Grinstein, Phys. Lett. B 256, 239 (1991).

[21] S. Herrlich and U. Nierste, Nucl. Phys. B455, 39 (1995).

[22] S. Nandi and H. n. Li, Phys. Rev. D 76, 034008 (2007).

[23] I. V. Musatov and A. V. Radyushkin, Phys. Rev. D 56, 2713 (1997).

[24] X. G. Wu and T. Huang, Phys. Rev. D 82, 034024 (2010).

[25] H. N. Li, Y. L. Shen, and Y. M. Wang, J. High Energy Phys. 01 (2014) 004.

[26] B. Aubert et al. (BABAR Collaboration), Phys. Rev. D 80, 052002 (2009).

[27] P. Masjuan, Phys. Rev. D 86, 094021 (2012).

[28] M. Hoferichter, B. Kubis, S. Leupold, F. Niecknig, and S. P. Schneider, Eur. Phys. J. C 74, 3180 (2014).

[29] A. Grardin, H. B. Meyer, and A. Nyffeler, Phys. Rev. D 94, 074507 (2016).

[30] A. V. Radyushkin, Phys. Rev. D 80, 094009 (2009).

[31] M. V. Polyakov, JETP Lett. 90, 228 (2009).
[32] S. S. Agaev, V. M. Braun, N. Offen, and F. A. Porkert, Phys. Rev. D 83, 054020 (2011).

[33] S. S. Agaev, V. M. Braun, N. Offen, and F. A. Porkert, Phys. Rev. D 86, 077504 (2012).

[34] P. Kroll, Eur. Phys. J. C 71, 1623 (2011).

[35] A. P. Bakulev, S. V. Mikhailov, and N. G. Stefanis, Phys. Rev. D 67, 074012 (2003).

[36] N. G. Stefanis, A. P. Bakulev, S. V. Mikhailov, and A. V. Pimikov, Phys. Rev. D 87, 094025 (2013).

[37] A. P. Bakulev, S. V. Mikhailov, A. V. Pimikov, and N. G. Stefanis, Phys. Rev. D 84, 034014 (2011).

[38] A. P. Bakulev, S. V. Mikhailov, A. V. Pimikov, and N. G. Stefanis, Phys. Rev. D 86, 031501 (2012).

[39] S. V. Mikhailov, A. V. Pimikov, and N. G. Stefanis, Phys. Rev. D 93, 114018 (2016).

[40] A. Khodjamirian, Eur. Phys. J. C 6, 477 (1999).

[41] P. Ball, V. M. Braun, and N. Kivel, Nucl. Phys. B649, 263 (2003).

[42] Y. M. Wang and Y. L. Shen, J. High Energy Phys. 05 (2018) 184.

[43] Y. L. Shen, Z. T. Zou, and Y. B. Wei, Phys. Rev. D 99, 016004 (2019).

[44] Y. L. Shen, Z. T. Zou, and Y. Li, arXiv:1901.05244.

[45] S. Ferrara, A. F. Grillo, G. Parisi, and R. Gatto, Phys. Lett. 38B, 333 (1972).

[46] I. I. Balitsky, V. M. Braun, and A. V. Kolesnichenko, Nucl. Phys. B312, 509 (1989).

[47] G. Duplancic, A. Khodjamirian, T. Mannel, B. Melic, and N. Offen, J. High Energy Phys. 04 (2008) 014.

[48] Y. M. Wang and Y. L. Shen, Nucl. Phys. B898, 563 (2015).

[49] Y. M. Wang, Y. B. Wei, Y. L. Shen, and C. D. Lü, J. High Energy Phys. 06 (2017) 062.

[50] V. L. Chernyak and A. R. Zhitnitsky, Nucl. Phys. B201, 492 (1982); B214, 547(E) (1983).

[51] A. P. Bakulev, S. V. Mikhailov, and N. G. Stefanis, Phys. Lett. B 508, 279 (2001); 590, 309(E) (2004).

[52] N. G. Stefanis, Phys. Lett. B 738, 483 (2014).

[53] A. Khodjamirian, T. Mannel, N. Offen, and Y.-M. Wang, Phys. Rev. D 83, 094031 (2011).

[54] S. J. Brodsky and G. F. de Teramond, Phys. Rev. D 77, 056007 (2008).

[55] J. Gronberg et al. (CLEO Collaboration), Phys. Rev. D 57, 33 (1998).

[56] S. Uehara et al. (Belle Collaboration), Phys. Rev. D 86, 092007 (2012).

[57] Y. S. Liu, W. Wang, J. Xu, Q. A. Zhang, S. Zhao, and Y. Zhao, arXiv:1810.10879.

[58] W. Wang, S. Zhao, and R. Zhu, Eur. Phys. J. C 78, 147 (2018). 